\title{
Novel Application of Pourbaix Diagrams to Model Precipitation in Upstream Biomanufacturing Process Solutions
}

\author{
Timothy Brantley ${ }^{1}$, Brandon Moore ${ }^{2}$, Chris Grinnell ${ }^{1}$, and Sarwat Khattak $^{1}$ \\ ${ }^{1}$ Biogen Inc \\ ${ }^{2}$ Biogen
}

April 3, 2021

\begin{abstract}
Commercial production of therapeutic proteins using mammalian cells requires complex process solutions, and consistency of these process solutions is critical to maintaining product titer and quality between batches. Inconsistencies between process solutions prepared at bench and commercial scale may be due to differences in mixing time, temperature, and $\mathrm{pH}$ which can lead to precipitation and subsequent removal via filtration of critical solution components such as trace metals. Pourbaix diagrams provide a useful tool to model the solubility of trace metals and were applied to troubleshoot the scale-up of nutrient feed preparation after inconsistencies in product titer were observed between bench- and manufacturing-scale batches. Pourbaix diagrams modeled the solubility of key metals in solution at various stages of the nutrient feed preparation and identified copper precipitation as the likely root cause of inconsistent media stability at commercial scale. Copper precipitation increased proportionally with temperature in bench-scale preparations of nutrient feed and temperature was identified as the root cause of copper precipitation at the commercial scale. Additionally, cell culture copper titration studies performed in bench-scale bioreactors linked copper-deficient mammalian cell culture to inconsistent titers at the commercial scale. Pourbaix diagrams can predict when trace metals are at risk of precipitating and can be used to mitigate risk during the scale-up of complex media preparations.
\end{abstract}

\section{Hosted file}

Cu Manuscript Draft_v2z-no figures.pdf available at https://authorea.com/users/405617/ articles/516553-novel-application-of-pourbaix-diagrams-to-model-precipitation-inupstream-biomanufacturing-process-solutions 
Pourbaix Diagram 1:

Prior to copper sulfate pentahydrate addition

$\mathrm{pH}:$ 9.2, Eh (V): -0.16

Pourbaix Diagram 2:

Copper sulfate pentahydrate and $\mathrm{NaOH}$ addition

pH: 9.6, Eh (V): -0.20

Pourbaix Diagram 3:

$\mathrm{HCl}$ addition

$\mathrm{pH}:$ 9.3, Eh (V): -0.23
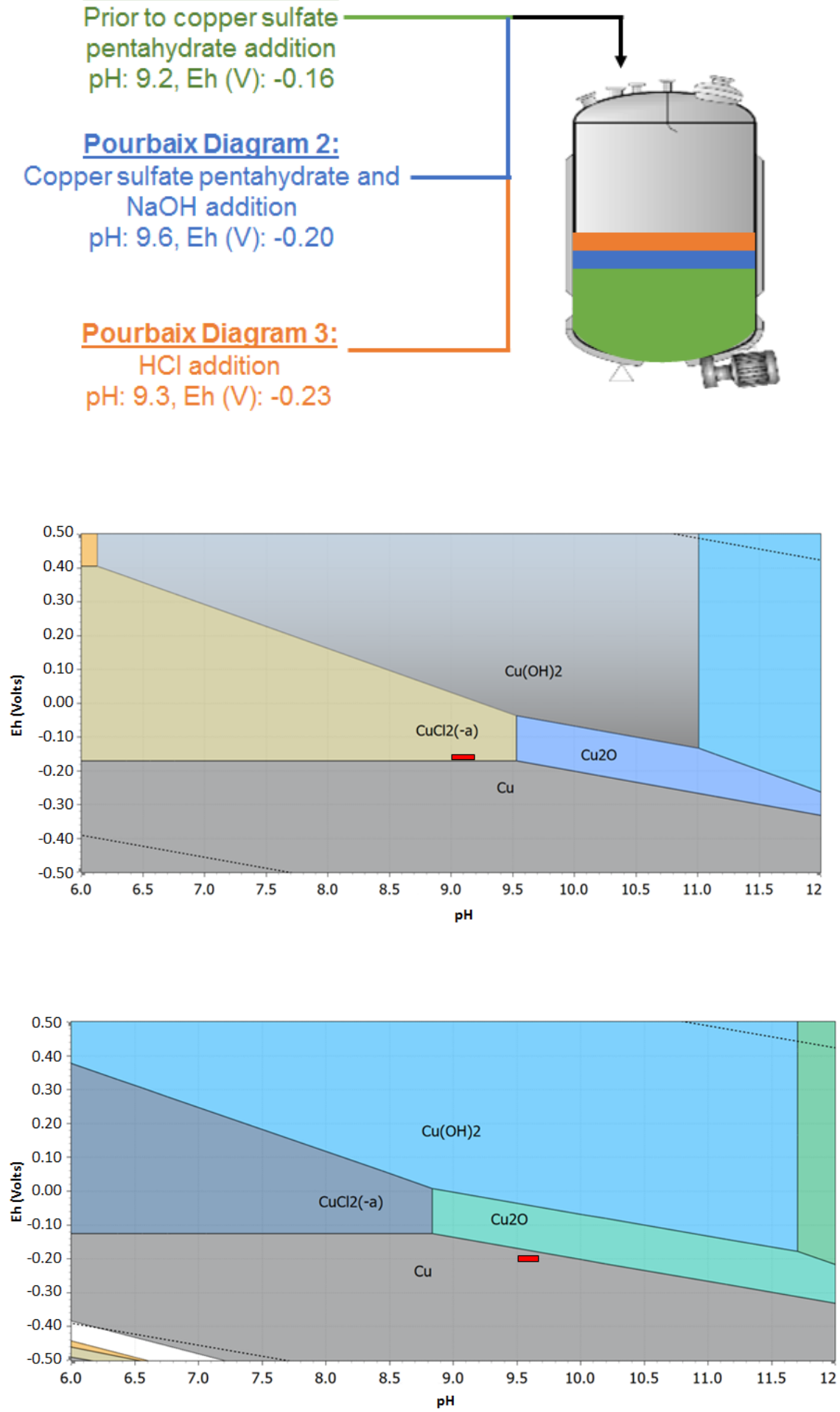

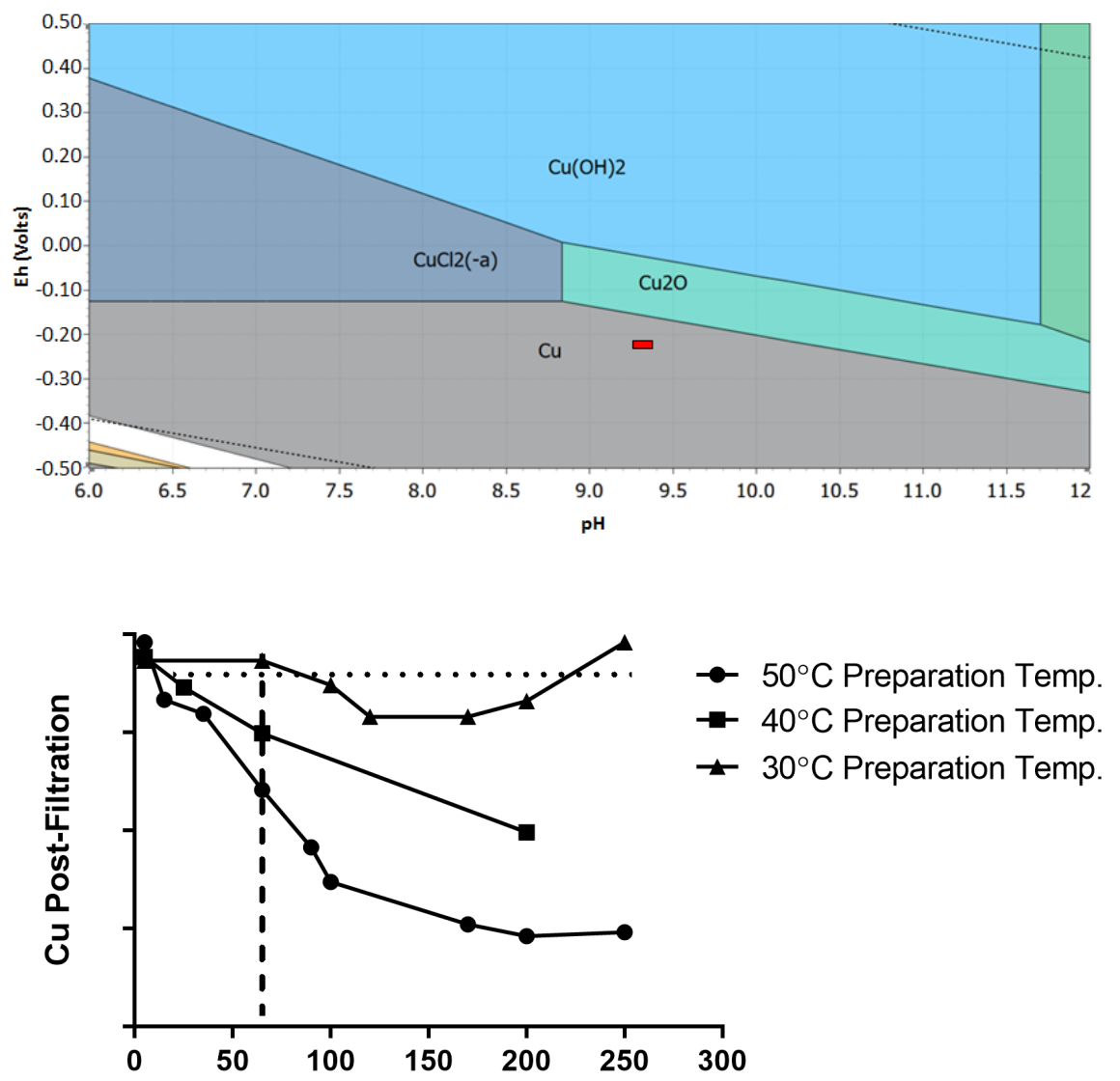

Time between cupric sulfate addition and filtration $(\mathrm{min})$ 

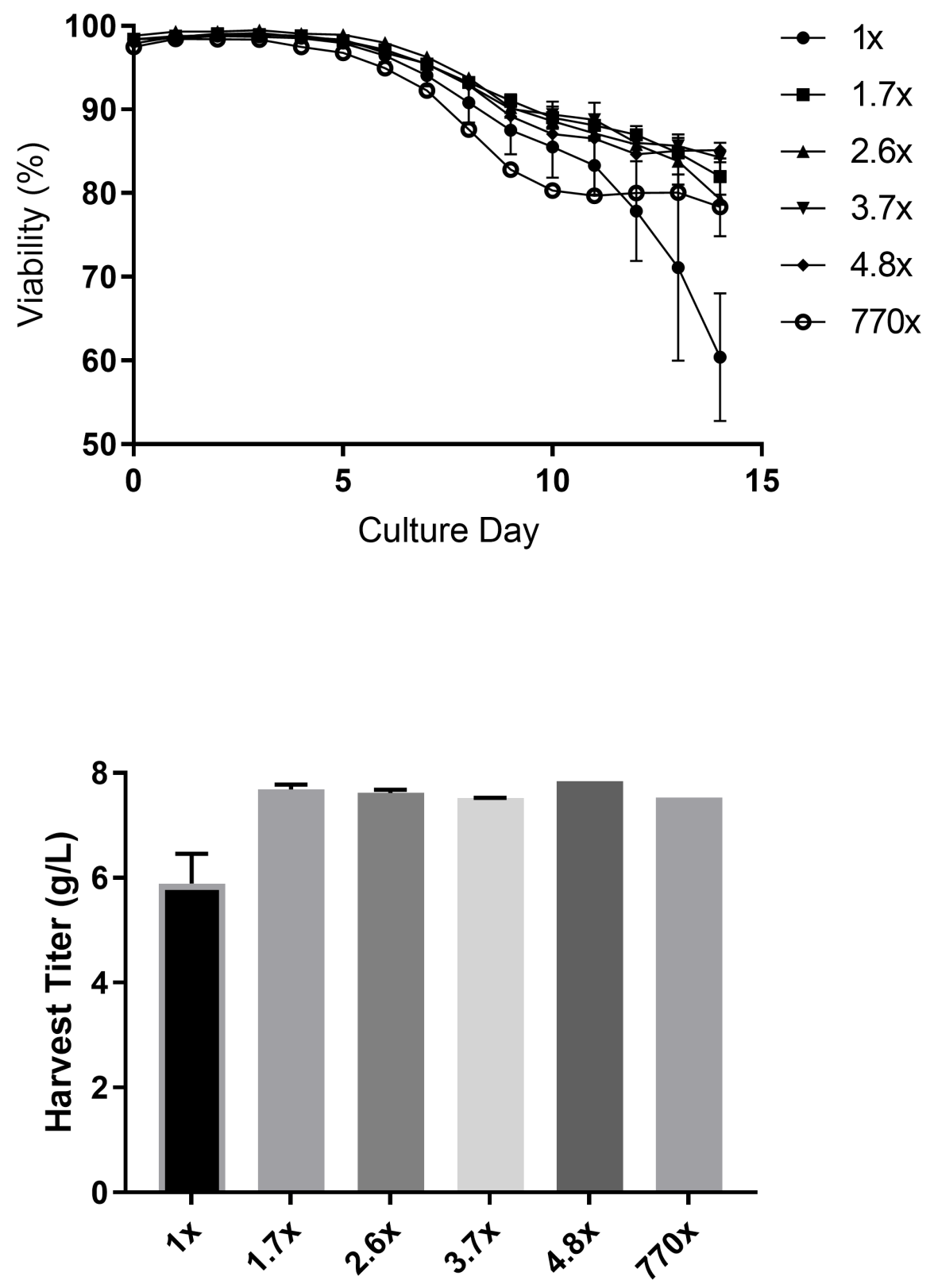

Relative Theoretical Harvest Copper Conc. 

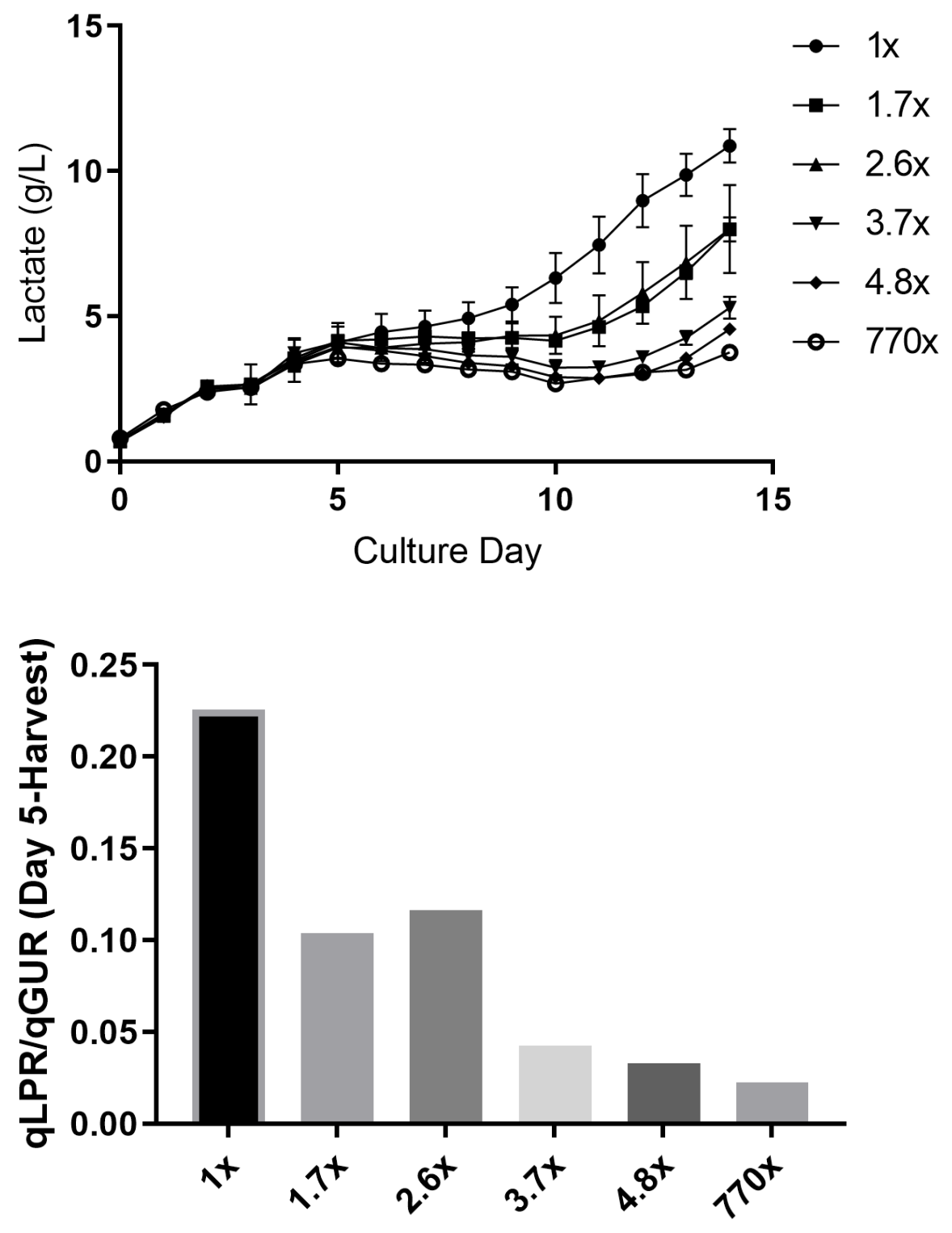

Estimated Copper Concentration at Harvest (nM) 


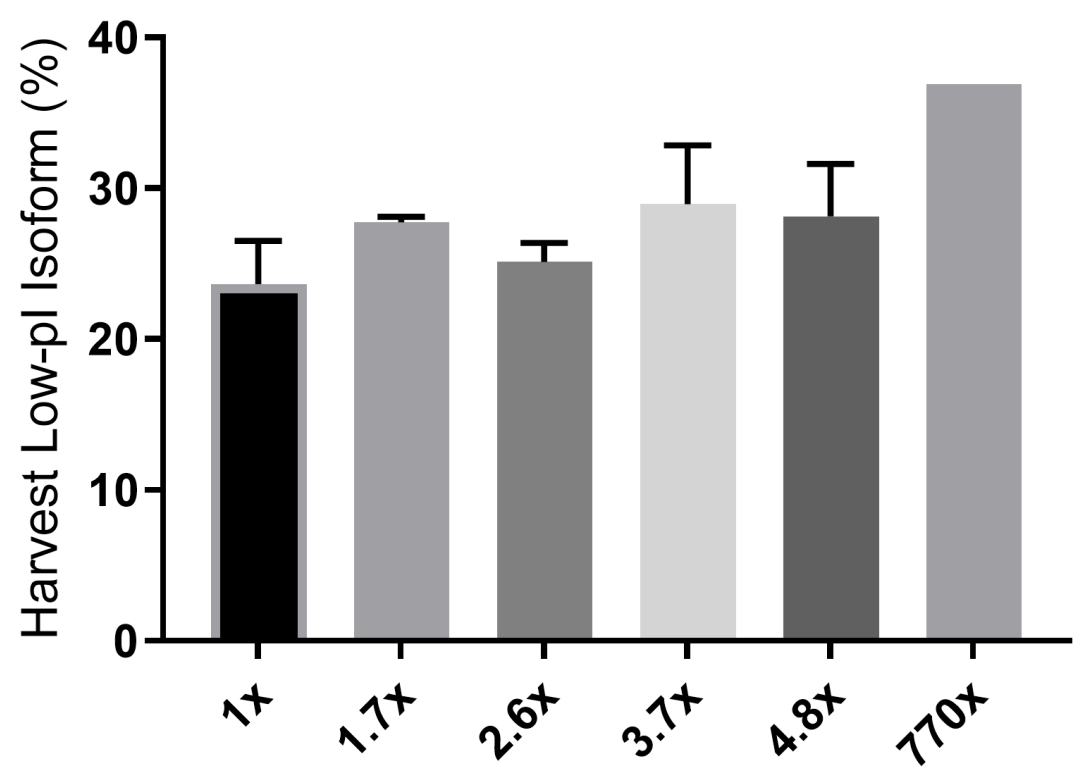

Relative Theoretical Harvest Copper Conc.

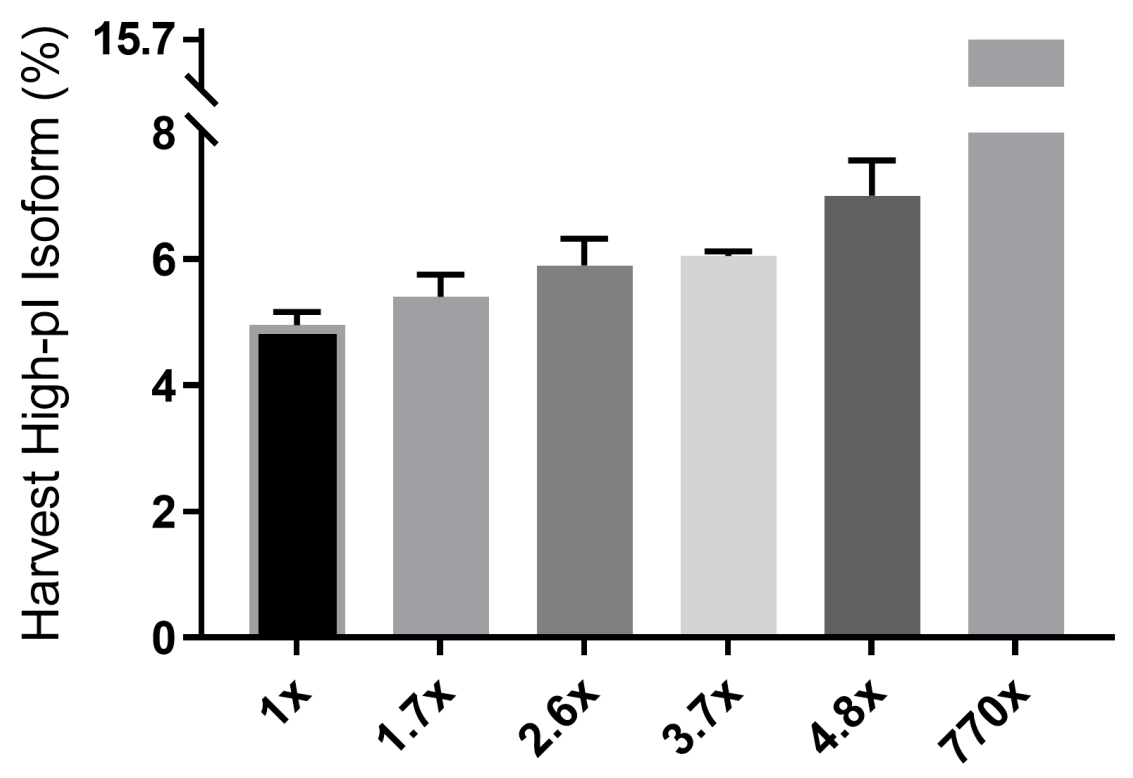

Relative Theoretical Harvest Copper Conc. 